\title{
Componentes de costo en los modelos de diseño de cadenas de abastecimiento
}

\author{
Fecha de recepción: 09/03/2010 - Aprobación:05/04/2010
}

\section{Oscar Javier Parra Ortega}

\section{Resumen}

La siguiente es una reseña sobre los costos asociados con la gestión de los sistemas logísticos en la actualidad. A continuación, se procederá a describir en detalle cada uno de estos costos, y a resaltar las particularidades de cada componente de costos en la toma de decisiones asociadas con el diseño de la red.

Finalmente, este artículo resalta las principales investigaciones relacionadas con el análisis de costos logísticos en el diseño de redes, así como su impacto en las utilidades en la cadena de abastecimiento.

\section{Abstract}

The following is a review of the costs associated to the current logistics management systems. Below, we proceed to describe in detail each of these costs, and highlight the characteristics of each costs component in decision making associated with the network design. Finally, this article highlights the main researches related to the analysis of logistic costs in network design and their impact on profits in the supply chain.

\section{Palabras clave}

Diseño de redes logísticas; costos logísticos; programación lineal entera-mixta.

\section{Key Words}

Supply Chain Design; Logistic Costs; Mixed Integer Linear Programming(MILP)

\section{Introducción}

La gestión de la cadena de abastecimiento por medio de la implementación de buenas prácticas logísticas, es una de las oportunidades de mejora identificadas en el proceso de autodiagnóstico logístico para empresas exportadoras realizado por Proexport, Logyca y Gs1, y cuyos resultados fueron divulgados en junio de 2009. A partir de esta necesidad, el diseño de toda cadena de suministro para las empresas colombianas debería asumirse como un esfuerzo compartido entre los actores que la conforman, para evitar duplicidades en la utilización de recursos y para generar sinergias.

Dado que el buen desempeño de las prácticas logísticas se basa en un correcto diseño de las redes de abastecimiento sobre 
las cuales se soportan todas las actividades de la cadena, es esencial identificar apropiadamente los costos logísticos que intervienen en dicha decisión. Cabe resaltar que actualmente un 50\% de las empresas exportadoras en Colombia sigue utilizando herramientas tradicionales de contabilidad para cuantificar sus costos logísticos, mientras que apenas un 10\% de ellas utiliza alguna metodología para analizar y discriminar sus costos de distribución, adquisición, gestión de inventarios y almacenaje.

\section{Costos logísticos}

\subsection{Costos por instalaciones}

Corresponden a los costos asociados con la localización y operación de la infraestructura física de la cadena de abastecimiento (Chopra, 2008). Se incurre en los costos de localización en la etapa de diseño de la red de distribución, y están asociados con decisiones que normalmente son difíciles de revertir. Los costos de operación, si bien son costos fijos, pueden variar de acuerdo con el nivel de utilización que se haga de la infraestructura instalada en la fase de diseño. Cabe anotar que este componente de costo varía en forma proporcional al número de instalaciones que componen la cadena de suministro.

\subsection{Costos por transporte}

De acuerdo con las decisiones de locali-

\section{Reseña de autor \\ Oscar Javier Parra Ortega}

(Politécnico Grancolombiano)

oparraor@poligran.edu.co

Ingeniero Industrial de la Universidad Industrial de Santander, docente investigador del Politécnico Grancolombiano, y miembro actual del Grupo de Investigación en Métodos Cuantitativos (GI-MEC) y del Centro de Investigación Colombia Regional y Empresarial (Cicre). zación y asignación (cuáles instalaciones atenderán a cada segmento de cliente y a través de qué medios de distribución), se incurre en unos costos de transporte. Esto hace que dichos costos también hagan parte esencial de las decisiones (y los modelos matemáticos asociados) de tipo estratégico. Los costos asociados con la entrega de productos hacia los clientes finales se clasifican como "outbound transport costs", mientras que los costos asocian dos con la recepción de insumos, materias primas y productos terminados, y su entrega por parte de los proveedores hacia los distintos actores de la cadena, se denominan "inbound transport costs". Son costos variables que se tienen en cuenta en la etapa de diseño de las redes de distribución.

\subsection{Costos por inventarios}

Son el componente crítico de costos en la fase de planeación y programación (mediano y corto plazo). No se tienen en cuenta en la etapa de diseño de la cadena, $y$ están ligados directamente con las políticas de reaprovisionamiento, las restricciones de espacio disponible en los puntos de venta, $y$ a las restricciones de capacidad por parte de los proveedores. Se dividen en: costos fijos por gestión de pedidos (costos por ordenar), y costos variables por adquisición y por almacenamiento de inventarios. Los costos por almacenamiento de inventarios incluyen, a su vez, los costos por obsolescencia, gestión de inventarios en bodega y el costo financiero del capital invertido en dichos inventarios. Cabe resaltar que los costos por inventarios corresponden a uno de los principales componentes del capital de trabajo de la cadena de suministro. A continuación se enuncian aspectos 
claves sobre el papel del capital de trabajo en el diseño y operación de las redes de distribución.

\subsection{Costos asociados con el capital neto de trabajo}

El capital de trabajo está conformado por los recursos que la cadena de abastecimiento requiere para poder operar. Corresponde a los activos corrientes (efectivo, inventarios y cartera), los cuales son utilizados para cubrir las necesidades de insumos, materia prima, y mano de obra a lo largo de toda la cadena de suministro. El capital de trabajo neto contable, corresponde entonces a la diferencia entre los activos corrientes y los pasivos corrientes de la cadena.

Dicho monto equivale a los recursos con los que cuenta la red de distribución para operar, si pagara todas sus obligaciones de corto plazo (Jones, KH.; Werner, ML.; et ál., 2001). En casos excepcionales, los pasivos corrientes superan a los activos corrientes, lo cual no implica que la empresa no pueda operar. Si el flujo de caja generado por los activos corrientes, no es suficiente para cubrir los pasivos corrientes, se presentaría iliquidez en alguno de los actores de la cadena de abastecimiento.

En el caso específico de las redes de distribución, los costos por inventarios, así como los costos de transporte y parte de los costos por instalaciones (los correspondientes al nivel de operación de la infraestructura instalada) se consideran parte esencial del capital de trabajo. El inventario disponible hace parte de los activos corrientes, mientras que los costos por gestión de inventarios, distribución y los costos fijos de operación de la infraestructura, harían parte de los pasivos corrientes.
Un modelo de diseño de redes, debe incluir todos estos componentes de costos, y de forma ideal, debería tener en cuenta una restricción asociada con mantener una relación preestablecida entre los activos y los pasivos corrientes (la cual se asocia con una cota inferior para la razón corriente de cada componente de la cadena de suministro). La razón corriente es la razón de liquidez que mide la capacidad de una compañía para cumplir con los pasivos a corto plazo (activos corrientes sobre pasivos corrientes).

\section{Análisis de costos logísticos}

Los costos logísticos encabezan el conjunto de medidas de desempeño utilizadas en la cadena de abastecimiento, tanto en el ámbito académico como empresarial.

Tal como resalta Scott Stevens, chief technology officer (СТО) para el Supply Chain Council,

Se estima que una reducción en un punto porcentual en los costos de su cadena de abastecimiento, puede ser equivalente a incrementar los beneficios entre 4 y 12 por ciento.

El SCC es un consorcio global sin ánimo de lucro, que desde 1996 ha venido estructurando diversas herramientas para comparar las actividades y el desempeño de cadenas de suministro a lo largo del mundo.

En los últimos años, algunos conceptos de la teoría en administración de costos han sido transferidos a la cadena de suministro, tales como el costeo basado en actividades (Kaplan, RS.; Cooper, R., 1998), o la administración de los objetivos de costos (Stefan, 2002). En la literatura disponible, los distintos autores difieren sobre la forma de clasificar los costos en la cadena de sumi-
Los costos logísticos encabezan el conjunto de medidas de desempeño utilizadas en la cadena de abastecimiento, tanto en el ámbito académico como empresarial. 
El objetivo a

seguir en la investigación en curso es enlazar estos costos con las decisiones estratégicas, deplaneación yoperativas, e incluirlos en su totalidad en un modelo de optimización, en el cual se diferencie claramente el capital de trabajo de los demás componentes de costo. nistro. Handfield (1998) clasifica los costos en la cadena de suministro en dos categorías: costos asociados con la gestión de productos, y los costos asociados a la interacción de los distintos actores que conforman la cadena. Seuring (1999: 18-23) divide los costos de la cadena de suministro en tres niveles diferentes: costos directos, costos basados en actividades y costos de transacción.

En la actualidad, de acuerdo con el desarrollo de la teoría de la cadena de suministro, los costos son una métrica, utilizada como soporte para la toma de decisiones y el análisis de beneficios, ya sea de una de las compañías que conforman a la cadena de suministro, o para la cadena en su totalidad. También se utiliza como un indicador de eficiencia y optimización de la cadena de suministro, razón por la cual se utiliza como función objetivo a minimizar.

La optimización en las cadenas de suministro está orientada a la minimización, tanto de los costos, como de los tiempos de ciclo. Whicker (2006) afirma que la relación entre los tiempos de ciclo y los costos, no es lineal. De acuerdo con el Modelo de Referencia de operaciones de la cadena de suministro (SCO, propuesto por la SCC como modelo de referencia para el desarrollo y mantenimiento de cadenas de abastecimiento), toda cadena de suministro está compuesta por cinco procesos básicos: "Source", "Make", "Deliver", "Return", y "Plan" (se mencionan en su idioma original por pertenecer a un modelo estándar).

El material y la información son recibidos de sus predecesores, a través del proceso source. Dentro del proceso make, dichos mate- riales son procesados junto con la información recibida para transformarlos en productos terminados. Estos productos se mueven a la siguiente etapa de la cadena en un proceso deliver, si no son descartados. Los productos restantes, no trasladados por alguna no conformidad, regresan a la cadena en un proceso return. Toda interacción entre dos procesos se considera un "enlace". Para coordinar estos procesos y sus enlaces, el modelo SCOR identifica un proceso "plan" en toda cadena de suministro.

Finalmente, cabe anotar que de acuerdo con el modelo SCOR (Tao-yong, 2008), los costos en la cadena de suministro se van acumulando de proceso en proceso, al mismo tiempo que se crea el valor agregado, y se clasifican en costos de: manufactura, transporte, inventario, transacción y relación en la cadena de suministro. De estos, los dos últimos son atribuibles a los enlaces entre procesos. El objetivo a seguir en la investigación en curso es enlazar estos costos con las decisiones estratégicas, de planeación y operativas, e incluirlos en su totalidad en un modelo de optimización, en el cual se diferencie claramente el capital de trabajo de los demás componentes de costo.

\section{Un modelo básico de minimización de los costos logísticos}

Con base en la descripción previa de los diversos componentes de costo en una cadena de abastecimiento, se enuncia a continuación una versión simplificada del modelo propuesto por Geoffrion (1974), para la localización y asignación de capacidades para una cadena de abastecimiento multietapa propuesto, en su versión mono producto. Sean: 
Índices:

$i=$ conjunto de proveedores de insumos de producción (Proveedores),

$j=$ conjunto de plantas de producción (Fabricantes),

$k=$ conjunto de puntos de distribución (Distribuidores),

$l=$ conjunto de mercados o puntos de demanda (Mercados),

$m=$ nivel de capacidad a utilizar por parte del fabricante, donde 1: capacidad fija, 2: capacidad flexible,

$n=$ nivel de capacidad a utilizar por parte del distribuidor, donde 1: capacidad fija, 2: capacidad flexible,

\section{Parámetros:}

$o=$ número de posibles localizaciones de proveedores de insumos de producción, $p=$ número de posibles localizaciones de plantas de producción, $q=$ número de posibles localizaciones de puntos de distribución, $r=$ número de mercados o puntos de demanda,

$C A P_{i}=$ capacidad estimada para cada proveedor $\mathrm{i}$, $C A P_{j m}=$ capacidad potencial del fabricante $\mathrm{j}$, operando al nivel $\mathrm{m}$, $C A P_{k n}=$ capacidad potencial del distribuidor $\mathrm{k}$, operando al nivel $\mathrm{n}$, $D_{l}=$ demanda anual del mercado $\mathrm{l}$, $f_{i}=$ costo fijo anualizado por utilizar al proveedor $\mathrm{i}$,

$f_{j m}=$ costo fijo anualizado por mantener en operación al fabricante $\mathrm{j}$ al nivel $\mathrm{m}$, $f_{k n}=$ costo fijo anualizado por utilizar al distribuidor $\mathrm{k}$ al nivel $\mathrm{n}$, $c_{i j}=$ costo de adquirir y enviar una unidad de insumo del proveedor $\mathrm{i}$ a la fábrica $\mathbf{j}$,

$c_{j k}=$ costo de producir un s.k.u. en la fábrica $\mathbf{j} \mathbf{y}$ enviarla al distribuidor $\mathbf{k},{ }^{1}$

$c_{k l}=$ costo de entregar un s.k.u. desde el distribuidor $\mathrm{k}$ al mercado $\mathrm{l}$,

$u_{j}=$ número de unidades de insumo requeridas para producir un s.k.u. en la fábrica $\mathrm{j}$,

Variables de decisión:

$y_{i}=1$ si se utiliza al proveedor $i, 0$ de lo contrario, $y_{j m}=1$ si la planta $j$ está abierta y opera al nivel $m, 0$ de lo contrario, $y_{k n}=1$ si el distribuidor $k$ está operando al nivel $n, 0$ de lo contrario, $x_{i j}=$ cantidad de insumo enviada desde el proveedor $i$ hacia la planta $j$, $x_{j k}=$ cantidad de s.k.u. enviadas desde el fabricante $j$ al distribuidor $k$, $x_{k l}=$ cantidad de s.k.u. enviadas desde el distribuidor $k$ al mercado $l$, 
Modelo de optimización:

Min

$$
\sum_{i=1}^{n} f_{i} y_{i}+\sum_{j=1}^{p} \sum_{m=1}^{2} f_{j m} y_{j m}+\sum_{k=1}^{q} \sum_{n=1}^{2} f_{k n} y_{k n}+\sum_{i=1}^{n} \sum_{j=1}^{m} c_{i j} x_{i j}+\sum_{j=1}^{n} \sum_{k=1}^{m} c_{j k} x_{j k}+\sum_{k=1}^{n} \sum_{l=1}^{m} c_{k l} x_{k l}
$$

Sujeto a

$$
\begin{gathered}
\sum_{j=1}^{p} x_{i j} \leq C A P_{i} * y_{i} \quad \forall i=1, \ldots, o \\
\sum_{k=1}^{q} x_{j k} \leq \sum_{m=1}^{2} C A P_{j m} * y_{j m} \quad \forall j=1, \ldots, p \\
\sum_{l=1}^{r} x_{k l} \leq \sum_{n=1}^{2} C A P_{k n} * y_{k n} \quad \forall k=1, \ldots, q \\
\sum_{k=1}^{n} x_{k l}=D_{l} \quad \forall j=1, \ldots, m \\
\sum_{j=1}^{p} x_{i j} \geq \sum_{k=1}^{q} u_{j} x_{j k} \quad \forall j=1, \ldots, p \\
\sum_{n=1}^{2} x_{k n} \leq 1 \quad \forall k=1, \ldots, q \\
\sum_{l=1}^{r} x_{k l} \quad \forall k=1, \ldots, q \\
\forall j=1, \ldots, p \\
\forall
\end{gathered} \quad \forall j
$$




$$
\begin{array}{rc}
y_{i} \in\{0,1\} & \forall i=1, \ldots, o \\
y_{j m} \in\{0,1\} & \forall j=1, \ldots, p, \forall m=1,2 \\
y_{k n} \in\{0,1\} & \forall k=1, \ldots, q, \forall n=1,2 \\
x_{i j} \geq 0 & \forall i=1, \ldots, o, j=1, \ldots, p \\
x_{j k} \geq 0 & \forall j=1, \ldots, p, j=1, \ldots, q \\
x_{k l} \geq 0 & \forall k=1, \ldots, q, j=1, \ldots, r
\end{array}
$$

Cabe resaltar, finalmente, la importancia de implementar este tipo de modelos para el diseño y rediseño de cadenas de abastecimiento, principalmente para pequeñas y medianas empresas, las cuales normalmente no tienen acceso a este tipo de herramientas, no las conocen, o no las aplican con regularidad.

\section{Bibliografía}

1. Ballou, RH. (2004). Business Logistics/supply management: planning, organizing and controlling the supply chain (5a. Ed.). Nueva York: Prentice Hall.

2. Chopra, S. (2008). Administración de la cadena de suministro: estrategia, planeación y operación (3a. Ed.). Nueva York: Prentice Hall.

3. Handfield, RB.; Nichols EL. (1998). Introduction to Supply Chain Management. Nueva Jersey: Prentice Hall.

4. Jones, KH.; Werner, ML.; et ál. (2001). Introducción a la contabilidad financiera (2da. Ed.). Nueva York: Prentice Hall.

5. Kaplan, RS.; Cooper, R. (1998). Cost \& Effect: Using Integrated Cost Systems to Drive Profitability and Performance. Boston, MA: Harvard Business School Press.

6. Seuring, S. (1999). Opportunities through cost management, the example of the supply chain for eco-products in the apparel industry. En Umweltwirtschaftsforum, 7, (4), 18-23.

7. Simchi-Levi, D.; Kaminsky, P.; y Simchi-Levi, E. (2003). Designing and managing the supply chain: concepts, strategies, and case studies (2da. Ed.). Nuueva York: Mc. Graw Hill.

8. Stefan S. (2002). Supply chain Target Costing An Apparel Industry Case Study. Cost Management in Supply Chain, 111.

9. Tao-Yong, S. (2008). Research on supply chain cost reduction based on process and time analysis. IEEE International Conference on Industrial Engineering and Engineering Management. IEEM.

10. Whicker L.; Bernon M.; Templar S.; Mena C. (2006). Understanding the relationships between time and cost to improve supply chain performance. Int. J. Production Economics, 6. 